
\title{
3 Research Square \\ The Effect of CCCP on Mitophagy in CD4+CD25+ Regulatory T Cells in Human Peripheral Blood
}

\section{Yu-Jie Yang}

Wuhan University

Md Rezaul Karim

Hubei University of Medicine https://orcid.org/0000-0002-8223-3486

Jang Yuan

Hubei University of Medicine

Xiao-Qian Peng

Hubei University of Medicine

Pei Zeng

Hubei University of Medicine

Yong Xu

Hubei University of Medicine

Zu-Neng Lu

Wuhan University

Yun-Fu Wang ( $\nabla$ wangyun@hbmu.edu.cn )

\section{Research}

Keywords: CCCP, Mitophagy, Regulatory T cells, Flow cytometry, fluorescence microscopy

Posted Date: July 7th, 2020

DOI: https://doi.org/10.21203/rs.3.rs-38918/v1

License: (c) (i) This work is licensed under a Creative Commons Attribution 4.0 International License.

Read Full License 


\section{Abstract}

Objective: To investigate the effects and mechanisms of different concentrations of CCCP on mitophagy in human peripheral blood regulatory T cells.

Methods: Tregs were isolated, identified, and then grouped, treating with CCCP at a concentration of 2.5 $\mu \mathrm{M}, 5 \mu \mathrm{M}, 10 \mu \mathrm{M}, 20 \mu \mathrm{M}$ and $40 \mu \mathrm{M}$ for $24 \mathrm{~h}$ in an incubator. Flow cytometry detected the reactive oxygen species (ROS), mitochondrial membrane potential (MMP), mitochondrial quality, and fluorescence microscopy observed the co-localization of mitochondria and lysosomes in each group.

Results: The purity of $\mathrm{CD} 4^{+} \mathrm{CD} 25^{+}$Tregs was $(93.36 \pm 1.87) \%$. With the increase of CCCP concentration, the ROS level gradually increased, while the MMP decreased gradually. About the mitochondria and lysosome fusion, the fluorescence intensity of orange (yellow) was the highest when the concentration of CCCP was in the range of 5-10 $\mu \mathrm{M}$ while decreased with the CCCP concentration continually increasing. The mitochondrial quality decreased with the increase of CCCP concentration. However, there was no significant difference between groups $\mathrm{C}, \mathrm{D}$ and $\mathrm{E}$. The mitochondrial quality of groups $\mathrm{F}$ and $\mathrm{G}$ were significantly lower than that of group $\mathrm{E}$.

Conclusions: With the concentration of CCCP gradually increased, the level of ROS in Treg cells increased, and MMP decreased, which promoted the mitophagy, mitochondrial quality maintains homeostasis. When ROS accumulated, and MMP decreased significantly, the mitophagy was inhibited, and the mitochondrial quality decreased significantly.

\section{Introduction}

Mitochondria are essential sites for eukaryotic cells to undergo bio-oxidation and energy conversion. Mitochondrial autophagy (Mitophagy) is a process of selectively scavenging excess or damaged mitochondria, which plays an essential role in regulating the number of mitochondria, maintaining mitochondrial function and the stability of the intracellular environment ${ }^{[1]}$. Mitophagy abnormalities can cause various diseases, such as cancer, autoimmune diseases, cardiovascular diseases, and metabolic syndrome. ${ }^{[2]}$. The carbonyl cyanide m-chlorophenylhydrazone (ССС) is an uncoupler of the mitochondrial oxidative respiratory chain, which can eliminate the transmembrane proton concentration difference on both sides of mitochondrial intima. It can slow down the process of oxidative phosphorylation driven by proton concentration difference and hinders the normal production of adenosine triphosphate (ATP). It also can make mitochondrial depolarisation to alter MMP and is thought to be induced parkin-dependent mitophagy to remove depolarised mitochondria, to maintain the stability of quality and quantity of mitochondria ${ }^{[3-4]}$. The treating time of CCCP is generally $24 \mathrm{~h}$, but for different cells, the optimal concentration for promoting autophagy is different ${ }^{[5]}$. Regulatory $T$ cells (Tregs) are $\mathrm{CD} 4^{+}$effector $\mathrm{T}$ cell subsets with immunosuppressive function; their phenotype is $\mathrm{CD} 4^{+} \mathrm{CD} 25^{+}$. Tregs can inhibit the activation of autoreactive $T$ cells and autoreactive $B$ cells, which is the key to maintaining immune balance and autoimmune tolerance ${ }^{[6]}$. The use of immunosuppressive Tregs for therapeutic 
purposes is of great interest to researchers because of their great potential in the treatment of

autoimmune diseases, prevention of transplant rejection, and tumor immunotherapy ${ }^{[7-9]}$. Previous research in our research group showed that the abnormal mitophagy in peripheral blood Treg cells in patients with myasthenia gravis $(M G){ }^{[10]}$. In this research, the autophagy state of mitochondria was changed In Vitro by using different CCCP concentrations in Tregs. The intracellular ROS, MMP, mitochondrial fusion, lysosome fusion, and mitochondrial quality level were observed. This was done to explore the effect and mechanism of CCCP on the mitophagy in Treg cells; to find the optimal concentration of CCCP to promote mitophagy in Treg cells. This can lay the foundation for our group to use CCCP in vitro to change the mitophagy status in Tregs of MG patients. This can also observe the level of autophagy and metabolism, and then explore the immune metabolic mechanism of abnormal mitophagy involved in $M G$ in the future.

\section{Materials And Methods}

\subsection{Materials}

Choosing twenty healthy volunteers in the medical examination center in our hospital, 10 males, 10 females, aged 24.3 - 49.6 years, mean age $(35.6 \pm 9.1)$ years old. Collecting $30 \mathrm{ml}$ peripheral blood from them for separating Tregs, the hospital ethics committee has reviewed the program, and volunteers have signed informed consent. Lymphocyte separation liquid was purchased from a Biotechnology Co., Ltd in China. Human $\mathrm{CD} 4^{+} \mathrm{CD} 25^{+}$Regulatory T cell sorting kit, LD column, and MS column were purchased from German company Miltemyi Biotec. Labeled antibodies FITC-CD4, PE-CD25 Purchased from BD Biosciences, USA, and DCFH-DA probe purchased from Sigma USA. JC-10 probe was purchased from Wuhan Plandor Biotechnology company, and Mito Tracker@Green, Lyso Tracker@Red were purchased from Life Technologies, USA. RPMI-1640 culture medium, fetal bovine serum (FBS), penicillin, and streptomycin were purchased from Gibco. A CytoFLEX analytical flow cytometer from Beckman Co., Ltd., and a fluorescence microscope of Olympus Corporation was also used.

\subsection{Methods}

\subsubsection{Isolation of PBMC and Treg Cells and Identification of Tregs}

(1) PBMC: Use a $50 \mathrm{ml}$ heparinized sterile syringe to collect $30 \mathrm{ml}$ peripheral blood, diluted to $90 \mathrm{ml}$ in a biological safety cabinet, and then added $15 \mathrm{ml}$ of lymphocyte separation solution to each of three sterile $50 \mathrm{ml}$ centrifuge tubes. After that, $30 \mathrm{ml}$ of diluted peripheral blood was slowly added along the tube wall and centrifuged at $2000 \mathrm{rpm} /$ minutes for 20 minutes. Cells from the white film layer were harvested and washed with PBS twice to obtain PBMC.

(2) $\mathrm{CD} 4^{+} \mathrm{CD} 25^{+}$Tregs: PBMCs were resuspended in $2 \mathrm{ml} \mathrm{PBS}$ and the cell count was approximately (4-5) $x 10^{7}$ cells/ml. The cells were centrifuged at $1200 \mathrm{rpm} /$ minutes for 8 minutes, and the supernatant was removed. After resuspension with buffer, appropriate amounts of $\mathrm{CD} 4^{+} \mathrm{T}$-cell biotinylated cocktail 
antibodies were added. After mixing, the cells were incubated at $4{ }^{\circ} \mathrm{C}$ in the dark for 5 minutes, and then the anti-biotin labeled beads were added and mixed. After incubation at $4{ }^{\circ} \mathrm{C}$ in the dark for 10 minutes, the cell suspension was passed through the LD column, and $C D 4^{+} T$ cells were isolated. Resuspended the obtained cells, add an appropriate amount of anti-CD25 magnetic beads antibody, mixed and incubated at $4{ }^{\circ} \mathrm{C}$ in the dark for 15 minutes, washed the cells with buffer, centrifuged, removed the supernatant, and resuspended. The cell suspension was passed through the MS column to obtain CD $4^{+} \mathrm{CD} 25^{+}$Tregs. Antibodies FITC-CD4, PE-CD25 labeled Tregs, the purity of which was detected by flow cytometry, and the cells were treated with CCCP at a concentration of $2.5 \mu \mathrm{M}, 5 \mu \mathrm{M}, 10 \mu \mathrm{M}, 20 \mu \mathrm{M}, 40 \mu \mathrm{M}$ respectively. The cells of each group were incubated for $24 \mathrm{~h}$ in a cell incubator at $37^{\circ} \mathrm{C}, 5 \% \mathrm{CO}_{2}$.

\subsubsection{Measurement of ROS}

Cells were collected in each group, and the blank group was resuspended with added $1 \mathrm{ml}$ serum-free cultures. The healthy control group and CCCP groups with different concentrations were added $1 \mathrm{ml} 10$ $\mu \mathrm{M}$ DCFH-DA active oxygen probe diluted in serum-free medium, incubated at $37^{\circ} \mathrm{C}$ in the dark for 20 minutes, and mixed every 3 minutes. Cells were washed three times with serum-free medium, resuspended in $200 \mu \mathrm{l}$ PBS, and measured by flow cytometry.

\subsubsection{MMP in Treg Cell}

The Treg cells in each group were collected, centrifuged, and the supernatant was removed and resuspended in $0.5 \mathrm{ml}$ serum-free medium. $1 \mathrm{ml}$ of serum-free medium was added to the blank group, and $1 \mathrm{ml}$ of diluted JC-10 MMP fluorescent probe was added to the healthy control group and CCCP groups. Incubated at $37^{\circ} \mathrm{C}$ in the dark for 20 minutes, centrifuge, remove the supernatant, wash with PBS twice, resuspended in $200 \mu \mathrm{l}$ of serum-free medium, and measured by flow cytometry.

\subsubsection{Observe the fusion of mitochondria and lysosomes}

Take $3 \mu$ mitochondrial probe $(1 \mu \mathrm{M})$ and $6 \mu$ lysosomal probe $(1 \mu \mathrm{M})$ into $6 \mathrm{ml}$ serum-free medium respectively, and pre-heat the probes at $37^{\circ} \mathrm{C}$ for 30 minutes. The cells in each group were collected, centrifuged, and the supernatant was removed. Then added $1 \mathrm{ml}$ of pre-heat mitochondrial probe working solution and $1 \mathrm{ml}$ of pre-heat lysosome probe working solution to the healthy control group and CCCP groups. , mixed, incubated at $37^{\circ} \mathrm{C}$ in the dark for 40 minutes, centrifuged, removed the supernatant, washed with PBS twice, resuspended with $50 \mu$ medium, and dropped it onto a slide, added coverslip and observed immediately under a fluorescence microscope. The images of mitochondrial green fluorescence and lysosomal red fluorescence were taken under the same magnification and the same field of vision. After Merging, the fusion of mitochondria and lysosomes was analyzed using Image $\mathrm{J}$ software.

\subsubsection{Determination of the mitochondrial quality level}

Take $3 \mu$ mitochondrial probe $(1 \mathrm{mM})$ into $6 \mathrm{ml}$ of serum-free medium-Preheat at $37^{\circ} \mathrm{C}$ for 30 minutes. Collect cells from each group, centrifuge, and the supernatant was removed, add $1 \mathrm{ml}$ of pre-heat 
mitochondrial probe working solution to each group. Mixed, incubated at $37^{\circ} \mathrm{C}$ in the dark for 40 minutes, centrifuged, and washed with PBS twice, resuspended in $200 \mu$ of serum-free medium, and measured by flow cytometry.

\subsection{Data Analysis}

SPSS 17.0 software was used for statistical analysis. Experimental data were presented as means \pm standard error of mean (SEM). Statistical comparisons were performed by using one-way analysis of variance (ANOVA). $P<0.05$ or less were considered to be of statistical significance.

\section{Results}

\subsection{Identification of Treg Cells}

The Treg cells separated by magnetic beads were labeled with FITC-CD4 and PE-CD25 antibodies, and the purity was $(93.36 \pm 1.87) \%$ (see figure 1$)$.

\subsection{ROS level in Treg Cells}

The DCFH-DA probe has no fluorescence and can pass through the cell membrane freely. After entering the cell, it is hydrolyzed into DCFH by intracellular esterase, while the DCFH cannot cross the cell membrane so that the probe can be easily loaded into the cell. ROS can oxidize non-fluorescent DCFH to fluorescent DCF, so the fluorescence of DCF can reflect the level of ROS in cells, the higher the fluorescence of DCF, the higher the level of ROS. Analysis of variance showed a significant difference between groups, $F=19.66, P<0.05$, ROS levels in each group were not equal; the linear effect between groups, $F=94.21, P<0.05$. Therefore, the level of ROS increased after CCCP treatment, and the level of ROS gradually increased as the concentration of CCCP increased, $P<0.05$, the difference was statistically significant (see figure 2).

\subsection{MMP in Treg Cell}

When the MMP is high, JC-10 accumulates in the matrix of mitochondria to form a polymer that can produce red fluorescence; when the MMP is low, JC-10 cannot accumulate in the matrix of mitochondria, at this time JC- 10 is a monomer that produces green fluorescence. Therefore, FITC and PE channels can be set for flow cytometry to represent green fluorescence and red fluorescence. The cross-sectional door is divided the two-dimensional scatter plot into four quadrants. The lower right quadrant represents that JC-10 exists as a monomer in the cell and emits green fluorescence. Comparing the proportion of cells in the lower right quadrant can reflect the level of MMP. The higher the proportion of cells in the lower right quadrant, the lower the MMP. Analysis of variance used for comparison between groups, $\mathrm{F}=$ 24.56, $P<0.05$, the difference was statistically significant; the total intergroup effect was broken down into linear and quadratic terms, the linear part $P<0.05$, statistically significant, quadratic item $P=0.711$, not statistically significant. Therefore, as the CСCP concentration gradually increased, the mitochondrial membrane potential gradually decreased (see figure 3 ). 


\subsection{Fusion of Mitochondria and Lysosomes in Treg Cells}

The mitochondria and lysosomes were labeled with probes, observed under the fluorescence microscope, the mitochondria showed green fluorescence, and the lysosomes showed red fluorescence. The mitochondrial autophagic lysosomes showed orange (or yellow) fluorescence after the fusion. The level of orange (yellow) fluorescence can reflect the level of mitochondrial autophagy in Treg cells. The stronger the orange (yellow) fluorescence, the higher the mitochondrial autophagy level. Analysis of variance used for comparison between groups, $\mathrm{F}=12.79, \mathrm{P}<0.05$, the difference was statistically significant; the fluorescence levels of cells in each group were different. The results of multiple comparisons showed that the fluorescence levels of the B, C, F, and G groups were lower than those of the E group, and the difference was statistically significant. Compared with the $G$ group, the $B, C, D, E$, and $F$ groups' fluorescence levels were higher. The difference was statistically significant, so we found that when the CCCP concentration was between 5-10 $\mu \mathrm{M}$, the orange (yellow) fluorescence level was the strongest, and as the CCCP concentration continued to increase, the orange (yellow) fluorescence level decreased, $\mathrm{P}<0.05$ (see figure 4 ).

\subsection{Mitochondrial Quality in Treg Cell}

The mitochondrial probe is a cellular osmotic structure derivative of carbonyl anthocyanin and is a bright green fluorescent probe. It almost does not fluoresce in aqueous solution, but only when it is in the lipid environment of mitochondria. Its ability to locate in the mitochondria is not affected by the MMP. Once it is ingested into the cells, they are not easy to be washed away. When the mitochondrial quality is impaired, the level of mitochondrial green fluorescence decreases. By standardizing cell number, dye concentration, and incubation time, semi-quantitative detection of mitochondrial quality can be achieved. Figure 5 shows the proportion of cells with standard mitochondrial quality on the V1R side, while the V1L site is the proportion of cells with impaired mitochondrial quality. The proportion of cells on the $\mathrm{V} 1 \mathrm{~L}$ side of each group was compared using analysis of variance (ANOVA), $F=94.36, P<0.05$. The difference was statistically significant, which means the mitochondrial quality of each group was not equal. The results of multiple comparisons showed no significant difference between $C, D$, and $E$ groups, $P>0.05$. Which means the mitochondrial quality of $C, D$ and $E$ groups maintained a dynamic balance; compared with group $\mathrm{E}$, the proportion of cells on the $\mathrm{V} 1 \mathrm{~L}$ side in group $\mathrm{B}$ was lower, while in the group $\mathrm{F}$ and the group $G$ increased, the difference was statistically significant $(P<0.05)$ (see figure 5).

\section{Discussions}

Mitochondria are organelles coated by two membranes present in most cells and have a diameter of about 0.5 to $10 \mu \mathrm{m}$. Mitochondria are the primary sites for intracellular oxidative phosphorylation and synthesis of adenosine triphosphate (ATP), and $95 \%$ of the energy required for cellular vitality comes from mitochondria. Therefore, it is also called a "power plant" for cells. In addition to supplying energy to cells, mitochondria also participate in cell differentiation, cell information transmission, and apoptosis, and can regulate cell growth and cell cycle ${ }^{[11]}$. Oxidative phosphorylation of mitochondria continues to 
provide energy support to the body, but ROS are produced during the process. ROS represents essential cell signaling molecules under the low or normal level and has a positive physiological effect, but high ROS levels can cause oxidative damage. When ROS increases, mitophagy can selectively remove damaged mitochondria, reduce the release of some pro-apoptotic factors. Thus, it prevents the intensification of oxidative stress and maintains the intracellular environment ${ }^{[12]}$. The mechanism may be: when the mTOR- dependent pathway sensed the increased environmental signal of ROS, its inhibitory effect on the ULK1-Atg13-FIP200 complex was weakened. And the complex was activated and transported from the cytoplasm to the specific site of the damaged mitochondrial membrane structure to form autophagy pronucleus, recruitment of downstream PI3K, LC3, and other autophagy-related proteins to form the autophagic vacuoles. Which was then followed by nucleation, autophagic body formation, and fused with the lysosome, and then mitophagy was completed. Abnormal mitochondria were removed timely, and cell survival was promoted ${ }^{[13]}$. Another mechanism that has been researched at present is the HIF-1a/BNIP3 / BNIP3L pathway, the increase of ROS promotes the expression of HIF-1a and HIF-1a activates the autophagy signal of downstream BNIP3/BNIP3L gene transcription. The expression of autophagy genes such as Beclin-1 and Atg5 are upregulated, eventually promoting mitophagy ${ }^{[14]}$. The mitochondrial permeability transition pore (mPTP) is a voltage-gated ion channel across the mitochondrial inner and outer membranes that consists of multiple subunits. It can regulate the permeability of the mitochondrial inner membrane. The increase of ROS level can promote the opening of mPTP, which activates mitophagy ${ }^{[15]}$.

Mitochondria are both major sources of oxidative stress and target organelles for oxidative stress. Mitochondria can sensitively sense the changes in oxygen concentration in the body. CCCP can affect the function of the electron transport chain during mitochondrial oxidative phosphorylation and inhibit ATP production, produce a lot of ROS. The accumulation of ROS can induce oxidative stress in cells by directly inducing single-stranded and double-stranded DNA breaks or by oxidizing fatty acids, amino acids in proteins, or enzymatic cofactors, and can directly attack mitochondria. Which is causing swelling of mitochondria and mitochondrial membranes permeability increases, MMP decreases, and apoptosisrelated proteins such as cytochrome $\mathrm{C}$ and apoptosis-inducing factors are released, which affect cell function and induce apoptosis. ${ }^{[16]}$. Studies have found that when the ROS produced by damaged mitochondria are released into the cytoplasm, which can damage the normal mitochondria around, and positive feedback produces a large amount of ROS that unbalances the mitochondrial oxidative phosphorylation and eventually leads to extensive mitochondrial damage ${ }^{[17]}$.

In the mitophagy process, autophagy body can wrap up damaged mitochondria in the early. In the midterm, mitochondrial autophagosomes fuse with lysosomes to form mature mitochondrial autophagy lysosomes ${ }^{[18]}$. Mitochondrial and lysosomes were labeled with Mito Tracker@Green and Lyso Tracker@Red, respectively. Under fluorescence microscopy, mitochondria showed green fluorescence, lysosomes showed red fluorescence, and mitochondrial autolysosomes showed orange (or yellow) fluorescence. Observing the level of orange (yellow) fluorescence can reflect the level of mitophagy in Treg cells. When the concentration of CCCP was between 5-10 $\mu \mathrm{M}$, the orange (yellow) fluorescence level 
was the strongest. As the CCCP concentration continued to increase, the orange (yellow) fluorescence level decreased. Which is indicating that the concentration of СССР was at 5-10 $\mu \mathrm{M}$, the level of mitophagy was the highest. As the concentration of CСCP continued to increase, the level of mitophagy decreased.

Mitochondria have a high degree of plasticity. Under normal circumstances, the number, morphology, and function of mitochondria are always maintained in a relatively stable state. When these conditions are disturbed, the energy supply of the cells and the life activities of the organism are bound to be affected. In order to deal with this damage, the cells have evolved a system of selective removal of damaged mitochondria - mitochondrial quality control (MQC), and mitophagy plays an essential role in the maintenance of mitochondrial quantity and quality by removing damaged mitochondria ${ }^{[19]}$. As can be seen from the above experiments, compared with the healthy control group, the mitochondrial quality decreased as CCCP's concentration increased. But the multiple comparisons between the groups found that there was no significant difference between the groups of $C, D, E$, and compared with the group $E$, the mitochondrial quality in group $\mathrm{F}$ and $\mathrm{G}$ decreased significantly. We can conclude that when the concentration of CCCP is between 2. 5-10 $\mu \mathrm{M}$, mitophagy can remove damaged mitochondria and synthesize new mitochondria through the reuse of degradation components. Mitochondrial quality maintained in a dynamic balance, but when the concentration of CCCP reaches $20 \mu \mathrm{M}$ and above, mitophagy cannot remove the damaged mitochondria adequately; mitochondrial quality decreased significantly.

\section{Conclusions}

As the concentration of CCCP gradually increases, the ROS level in Treg cells gradually increases, and the MMP gradually decreases, but only when the ROS level slightly increases. The MMP slightly decreases, mitophagy is promoting, mitochondrial quality maintains a dynamic balance. When ROS accumulates, and MMP decreases significantly, a large number of mitochondria are damaged, the mitophagy level decreases, and mitochondrial quality is severely damaged. Mitochondria are the most critical organelle involved in energy metabolism in Treg cells. The proliferation and differentiation of Treg cells are mainly dependent on mitochondrial lipid oxidation to supply energy and the proportion occupied by aerobic glycolysis a little ${ }^{[20]}$. When the mitochondrial quality of Treg cells is severely damaged, the energy metabolism of Treg cells may be disturbed. The signal transduction of cells and the production of free radicals are abnormal, which may induce cell apoptosis, the specific mechanisms need to be further study.

\section{Declarations}

\section{Ethical Approval and Consent to participate}

The study was approved by the hospital ethics committee, Taihe Hospital, Hubei University of Medicine, Shiyan, Hubei, China. The purpose of this research was explained to the participant. Once the verbal 
consent was understood and agreed, a written consent was then obtained from the participant.

\section{Consent for publication}

All the authors agreed to publish this work.

\section{Availability of data and materials}

Available on request to the corresponding author.

\section{Competing interests}

None to declare.

\section{Funding}

National Natural Science Foundation of China (NO: 81671238); Hubei University of Medicine Outstanding Young and Middle-aged Science and Technology Innovation Team Project (NO:2014CXX01).

\section{Authors' contributions}

All authors contributed equally to this work in different phases of this project.

\section{Acknowledgements}

We much appreciate Taihe Hospital, Hubei University of Medicine and funding bodies for their help.

\section{Authors' information}

Yu-Jie Yang: Attending physician, Department of Neurology, Renmin Hospital, Wuhan University, Wuhan, Hubei, China.

Md Rezaul Karim: Principal Investigator, Hubei Key Laboratory of Embryonic Stem Cell Research, Institute of Neuroscience, Hubei University of Medicine, Shiyan, Hubei, China.

Jang Yuan: Attending physician, Department of Neurology, Taihe Hospital, Hubei University of Medicine, Shiyan, Hubei, China.

Xiao-Qian Peng: Attending physician, Department of Neurology, Taihe Hospital, Hubei University of Medicine, Shiyan, Hubei, China.

Pei Zeng: Attending physician, Department of Neurology, Taihe Hospital, Hubei University of Medicine, Shiyan, Hubei, China.

Yong Xu: Resident physician, Department of Neurology, Taihe Hospital, Hubei University of Medicine, Shiyan, Hubei, China. 
Zu-Neng Lu: Chief physician, Department of Neurology, Renmin Hospital, Wuhan University, Wuhan, Hubei, China.

Yun-Fu Wang: Chief physician and Director, Department of Neurology, Taihe Hospital, Hubei University of Medicine, Shiyan, Hubei, China.

\section{References}

1. Ni HM, Williams JA, Ding WX. Mitochondrial dynamics and mitochondrial quality control[J]. Redox Biology. 2015;4(C):6-13.

2. Jiang P, Mizushima N. Autophagy and human diseases[J]. Cell Res. 2014;24(1):69-79.

3. Kane MS, Paris A, Codron P, et al. Current mechanistic insights into the CCCP-induced cell survival response[J]. Biochem Pharmacol. 2017;148:100-10.

4. Kwon KY, Viollet B, Yoo OJ. CCCP induces autophagy in an AMPK-independent manner[J]. Biochemical Biophysical Research Communications. 2011;416(3-4):343-8.

5. Park YS, Choi SE, Koh HC. PGAM5 regulates PINK1/Parkin-mediated mitophagy via DRP1 in CCCPinduced mitochondrial dysfunction[J]. Toxicol Lett. 2017;284:120-8.

6. Qiao YC, Pan YH, Ling W, et al. The Yin and Yang of regulatory $T$ cell and therapy progress in autoimmune disease.[J]. Autoimmun Rev. 2017;16(10):1058-70.

7. Spence A, Klementowicz JE, Bluestone JA, et al. Targeting Treg signaling for the treatment of autoimmune diseases[J]. Curr Opin Immunol. 2015;37:11-20.

8. Li J, Lai X, Liao W, et al. The dynamic changes of Th17/Treg cytokines in rat liver transplant rejection and tolerance[J]. Int Immunopharmacol. 2011;11(8):962.

9. Wang H, Franco F, Ho PC. Metabolic Regulation of Tregs in Cancer: Opportunities for Immunotherapy. [J]. Trends in Cancer. 2017;3(8):583.

10. Wang N, Zou L, Yuan J, et al. The abnormal mitophagy of regulatory T cells in peripheral blood of patients with myasthenia gravis[J]. Chinese Journal of Neuroimmunology \& Neurology,2017.

11. Picard M, Mcewen BS, Epel E, et al. An Energetic View of Stress: Focus on Mitochondria.[J]. Frontiers in Neuroendocrinology, 2018.

12. Fang $C$, Gu L, Smerin D, et al. The Interrelation between Reactive Oxygen Species and Autophagy in Neurological Disorders:[J]. Oxidative Medicine \& Cellular Longevity, 2017, 2017(3):1-16.

13. Wible DJ, Bratton SB. Reciprocity in ROS and autophagic signaling[J]. Curr Opin Toxicol. 2018;7:2836.

14. Rani V, Deep G, Singh RK, et al. Oxidative stress and metabolic disorders: Pathogenesis and therapeutic strategies[J]. Life Sci. 2016;148(11):183-93.

15. Murrow L, Debnath J. Autophagy as a stress-response and quality-control mechanism: implications for cell injury and human disease.[J]. Annual Review of Pathology Mechanisms of Disease. 2013;8(8):105-37. 
16. Chen YF, Liu H, Luo XJ, et al. The roles of reactive oxygen species (ROS) and autophagy in the survival and death of leukemia cells.[J]. Crit Rev Oncol Hematol. 2017;112:21-30.

17. Watanabe R, Fujii $\mathrm{H}$, Shirai $\mathrm{T}$, et al. autophagy plays a protective role as an anti-oxidant system in human T cells and represents a novel strategy for induction of T-cell apoptosis[J]. Eur J Immunol. 2014;44(8):2508-20.

18. Patergnani S, Pinton P. Mitophagy and mitochondrial balance. Methods Mol Biol. 2015;1241:18194.

19. Pickle S, Vigie P, Youle RJ. Mitophagy and Quality Control Mechanisms in Mitochondrial Maintenance[J]. Curr Biol. 2018;28(4):R170-85.

20. Michalek RD, Gerriets VA, Jacobs SR, et al. Cutting edge: distinct glycolytic and lipid oxidative metabolic programs are essential for effector and regulatory CD4 + T cell subsets.[J]. J Immunol. 2011;186(6):3299-303.

\section{Figures}
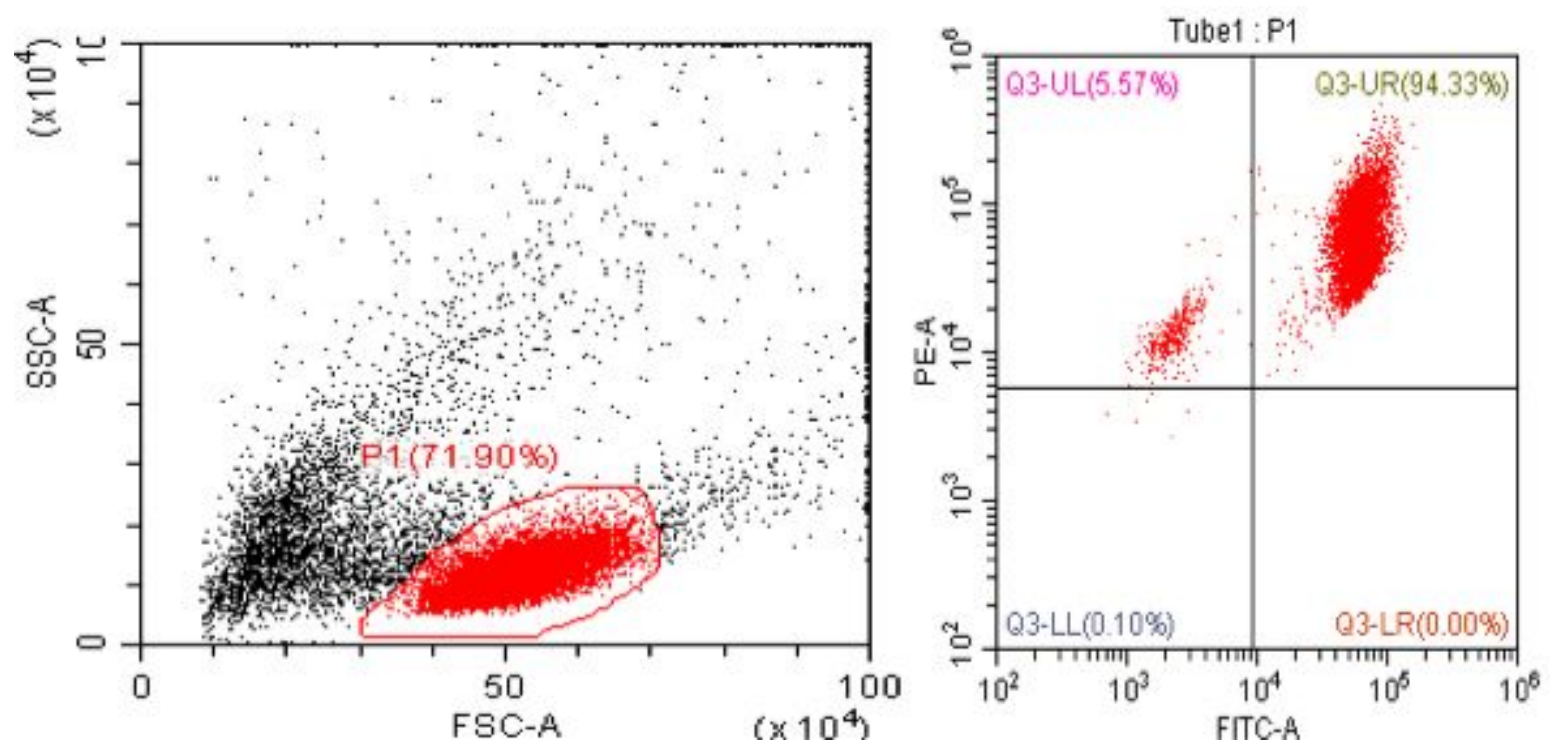

Figure 1

FITC-CD4, PE-CD25 labeled Tregs (target cells in P1, a proportion of CD4+CD25+ cell in the Q3-UR quadrant). 


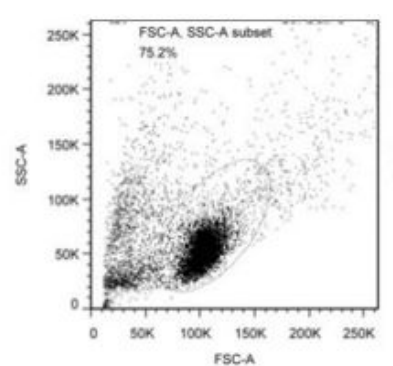

$\mathrm{A}_{0}$

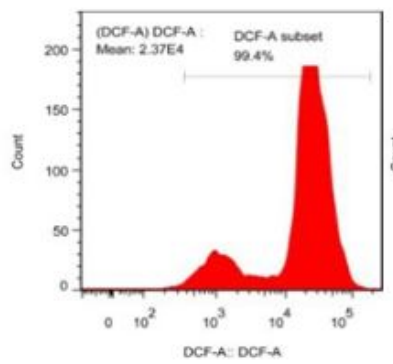

C

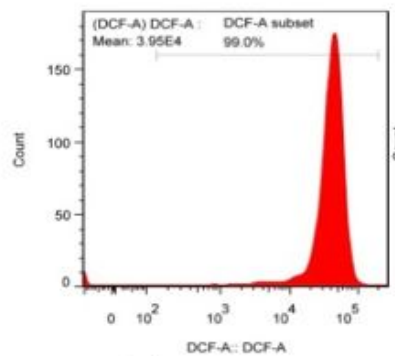

F

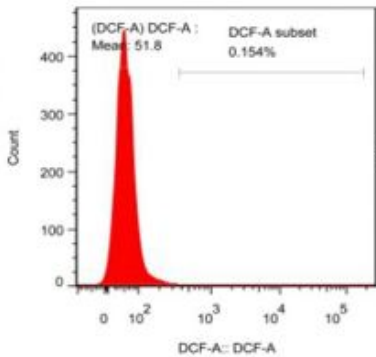

A

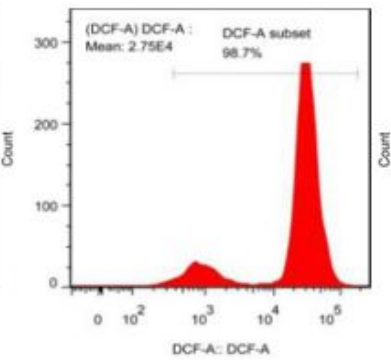

D

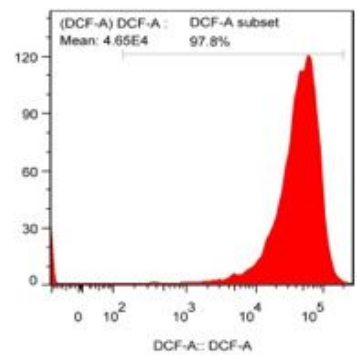

G

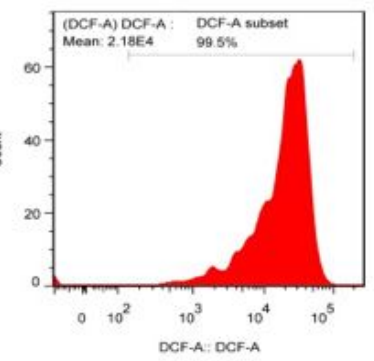

B

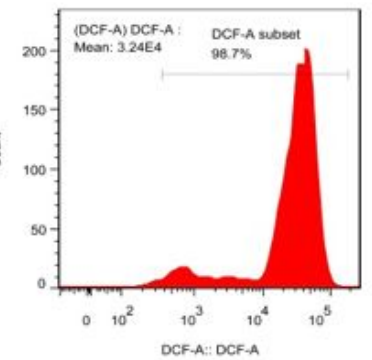

E

FITC

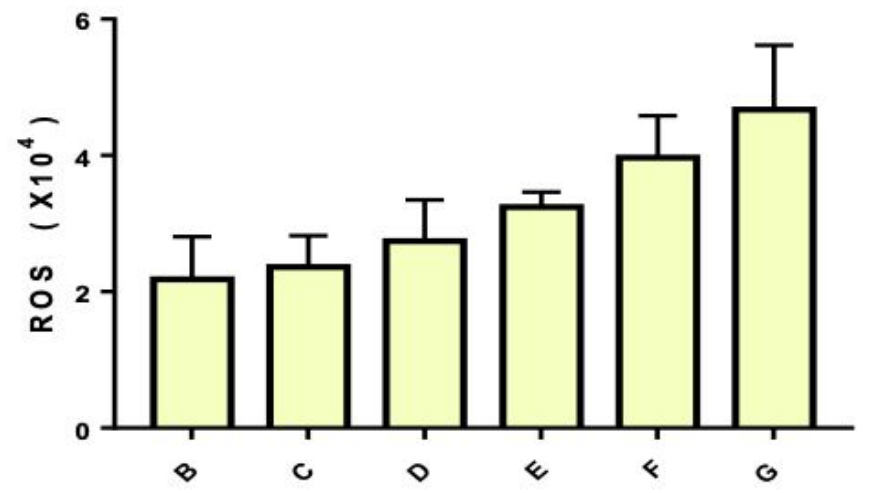

Figure 2

Detection of ROS level in each group by DCFH-DA probe, the average fluorescence intensity of DCF represents ROS levels (A. blank group, unstained Tregs; B. healthy control group; C. CCCP $2.5 \mu \mathrm{M}$; D. CCCP $5.0 \mu \mathrm{M}$; E. ССCP $10 \mu \mathrm{M} ;$ F. СCCP $20 \mu \mathrm{M} ;$ G. CCCP $40 \mu \mathrm{M})$. 


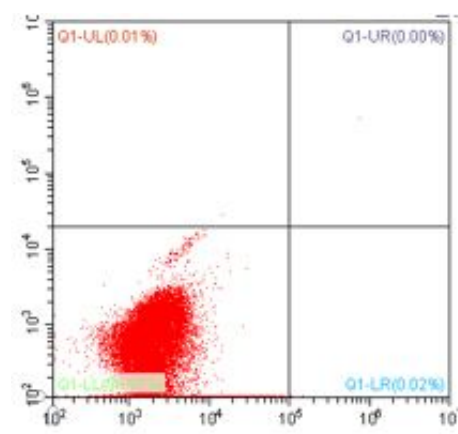

A
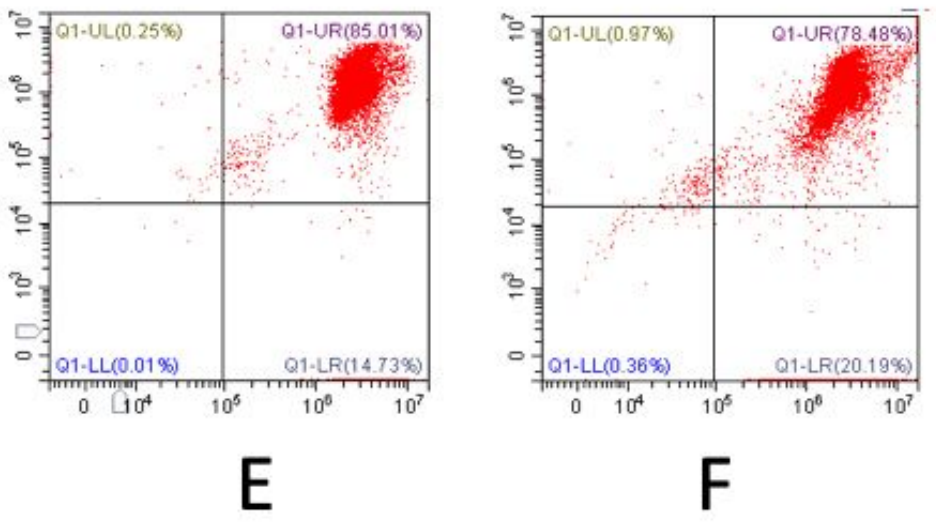

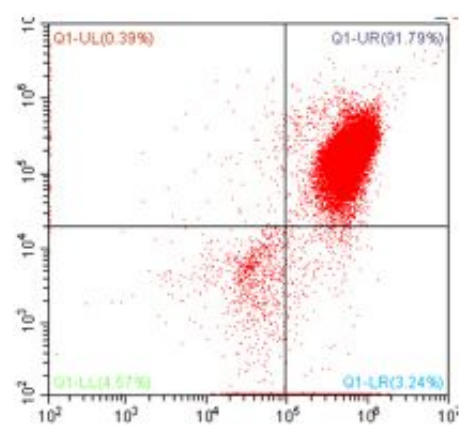

C

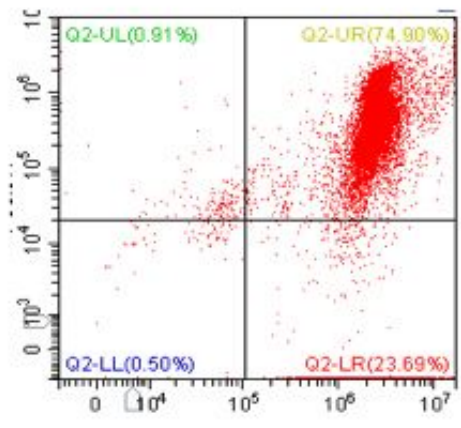

G

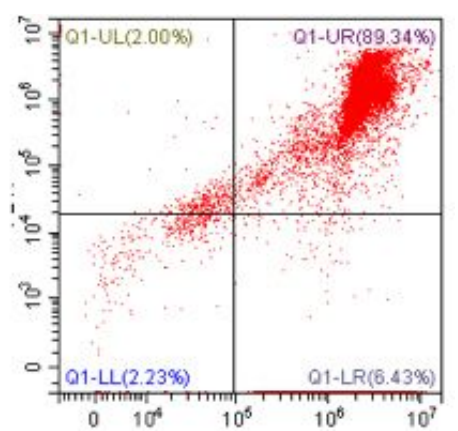

D

\begin{tabular}{lrrrr}
\hline Group & \multicolumn{2}{c}{ Right lower quadrant cell ratio $(\%)$} & F Value & P Value \\
\hline B & $0.68 \pm 0.55$ & Cluster & 24.56 & 0.000 \\
C & $3.32 \pm 2.10$ & 1inear items comparison & 119.80 & 0.000 \\
D & $7.10 \pm 2.78$ & Deviation & 0.749 & 0.564 \\
E & $14.35 \pm 6.39$ & Second items comparison & 0.139 & 0.711 \\
F & $20.15 \pm 6.78$ & Deviation & 0.952 & 0.424 \\
G & $22.39 \pm 7.70$ & & & \\
\hline
\end{tabular}

Figure 3

Detection of MMP level in each group by JC-10 probe, the proportion of cells in the lower right quadrant indicates the level of MMP (A. blank group, unstained Tregs; B healthy control group; C. CCCP $2.5 \mu \mathrm{M} ; \mathrm{D}$. CCCP $5.0 \mu \mathrm{M}$; E. CCCP $10 \mu \mathrm{M}$; F. CCCP $20 \mu \mathrm{M}$; G. CCCP $40 \mu \mathrm{M}$ ). 


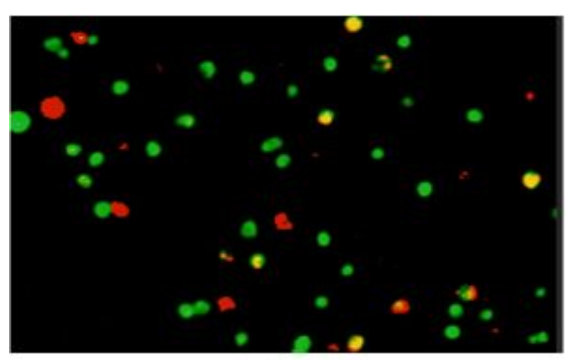

B

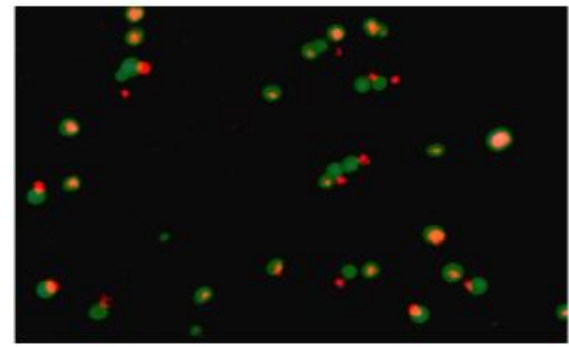

D

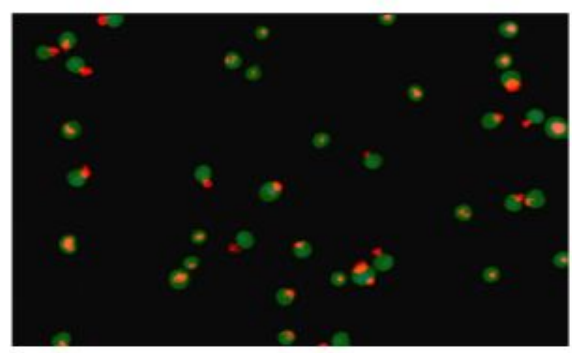

$\mathrm{F}$

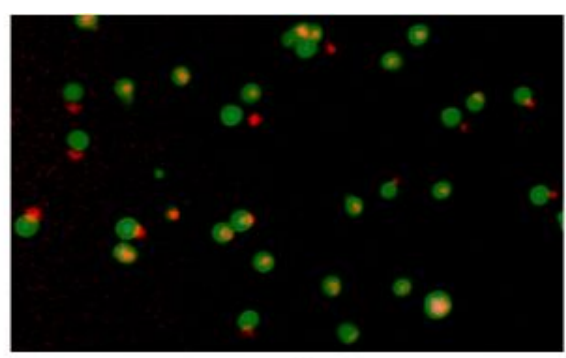

C

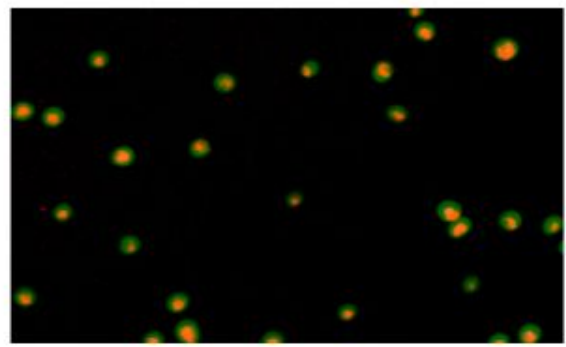

$E$

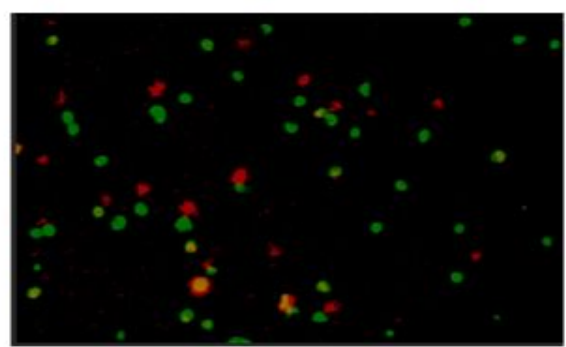

G

Mitochondrial quality levels

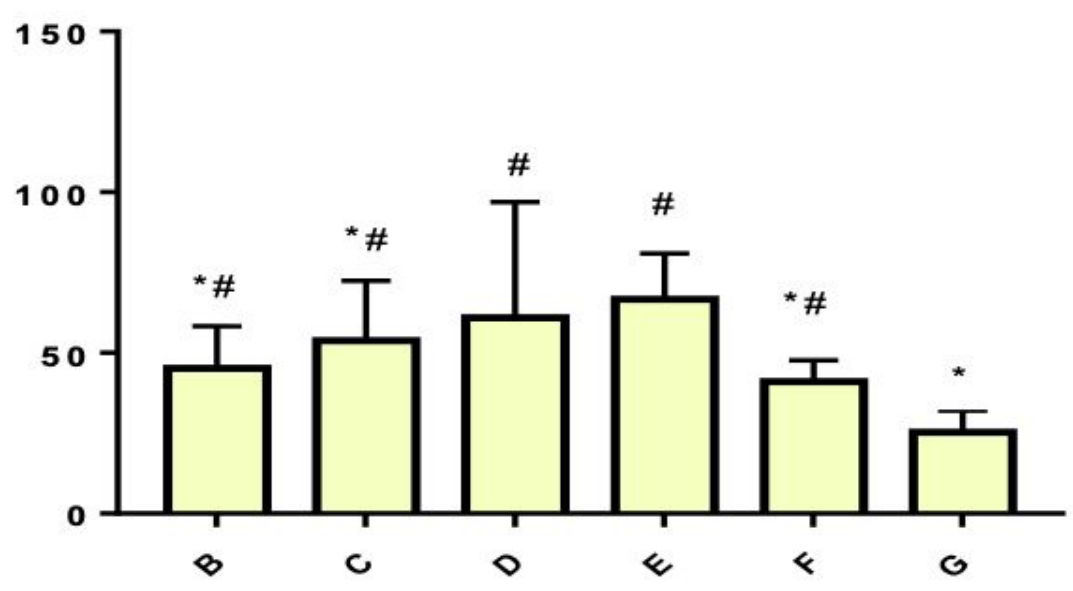

Figure 4

mitochondria and lysosomes were labeled with probes, observed the yellow (orange) fluorescence levels in each group (the fluorescence level was analyzed with Image $\mathrm{J}$ software, magnification was: 200X; B. healthy control group; C. CCCP $2.5 \mu \mathrm{M}$; D. CCCP $5.0 \mu \mathrm{M}$; E. CCCP $10 \mu \mathrm{M}$; F. CCCP $20 \mu \mathrm{M}$; G. CCCP $40 \mu \mathrm{M}$, compared with CCCP $10 \mu \mathrm{M}$ group, ${ }^{\star} \mathrm{P}<0.05$; compared with CCCP $40 \mu \mathrm{M}$ group, \# $\mathrm{P}<0.05$ ). 


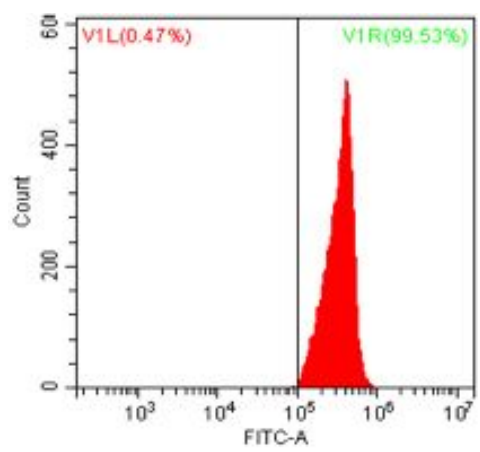

B

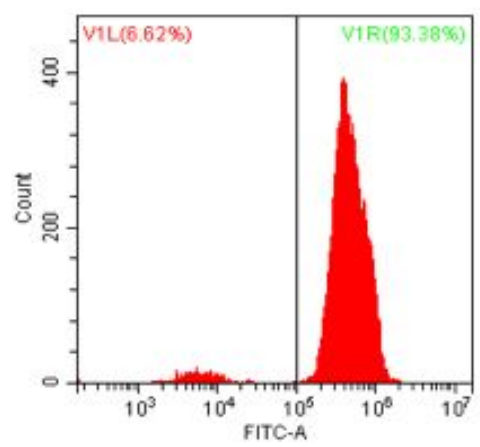

$\mathrm{E}$

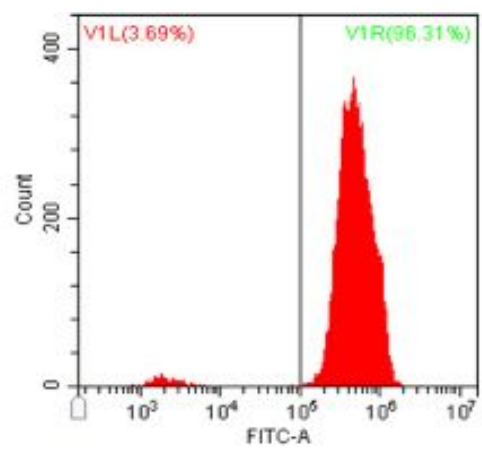

C

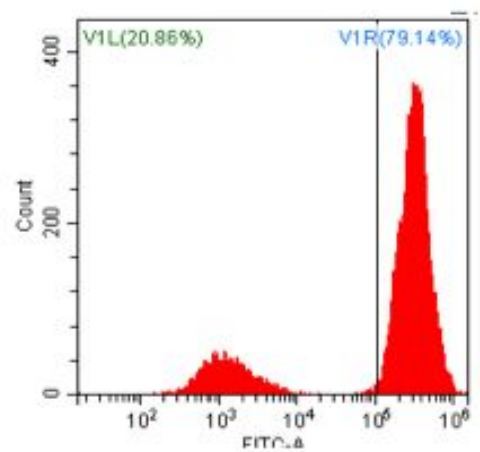

$\mathrm{F}$

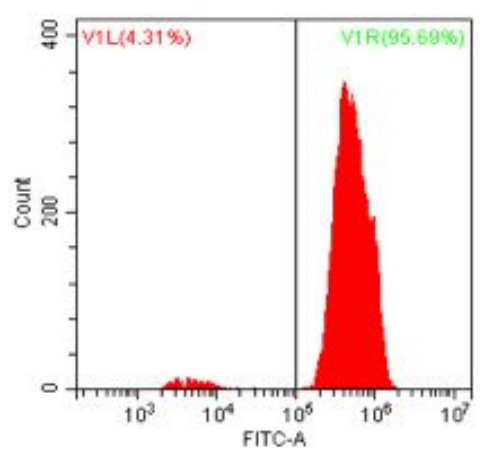

D

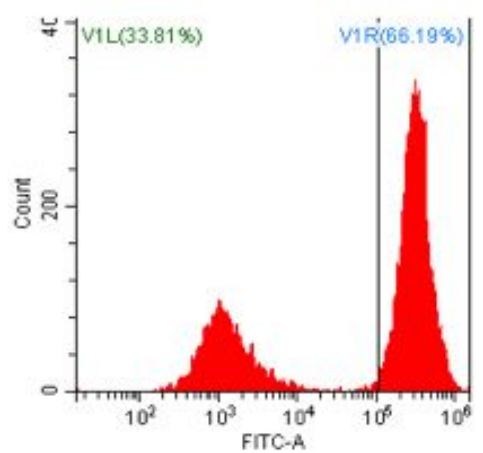

G

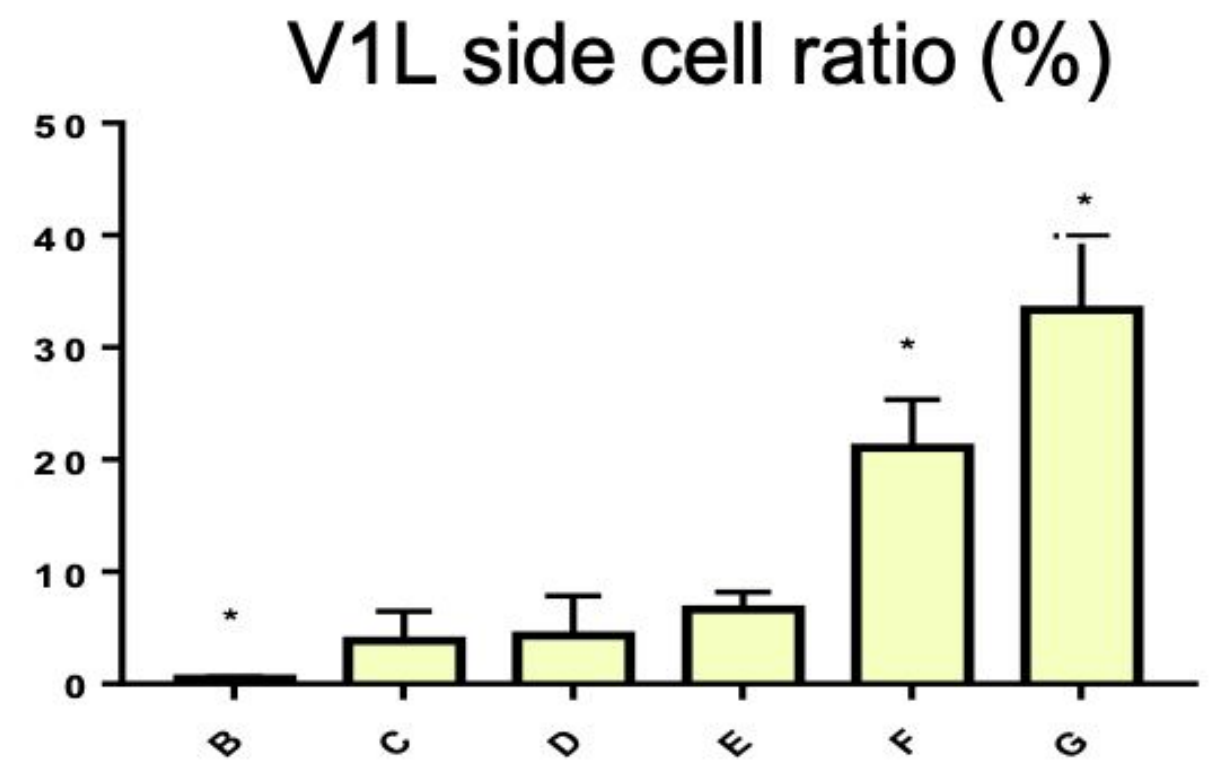

Figure 5

Probe labeled mitochondria, flow cytometry detected mitochondrial quality levels in each group (B. healthy control group; C. CCCP $2.5 \mu \mathrm{M}$; D. CCCP $5.0 \mu \mathrm{M}$; E. CCCP $10 \mu \mathrm{M}$; F. CCCP $20 \mu \mathrm{M}$; G. CCCP $40 \mu \mathrm{M}$; compared with CCCP $10 \mu \mathrm{M}$ group, $\left.{ }^{*} \mathrm{P}<0.05\right)$. 